\title{
METAL-SEMICONDUCTOR HETEROJUNCTION ROLE IN CdTe DETECTORS
}

\author{
Lubomír GRMELA, Ondrej ŠIK \\ Department of Physics, Faculty of Electrical Engineering and Communication, \\ Brno University of Technology, Technicka 8, 61600 Brno, Czech Republic, tel: +420 541143 256, \\ e-mail: grmela@feec.vutbr.cz
}

\begin{abstract}
We have performed noise spectroscopy and charge transport properties analysis of CdTe detectors. Two types of high volume detectors are compared: Low-ohmic (based on low-resistivity crystal material $\rho=70 \Omega \mathrm{cm}$ ) and semi-insulating $\left(\rho=10^{8} \Omega \mathrm{cm}\right)$.The theoretical fundaments of contacts role in detector system are given. We observed high asymmetry of IV characteristics of the lowohmic sample between "normal" and "reverse" bias, showing improper quality of contacts preparation, caused by higher concentration of impurities in metal-semiconductor area. This finding is supported by the fact that the low frequency noise spectral density is proportional to applied voltage with exponent 2.7, which is higher than the theoretical value 2.The semi-insulating sample very good contact rectification effect symmetry and less additive noise to the detector system.
\end{abstract}

Keywords: Cadmium Telluride, 1/f low frequency noise, semiconductor reliability, semiconductors charge transport mechanism

\section{INTRODUCTION}

Cadmium-Telluride (CdTe) based radiation detectors are based on semiconductor ionization chamber principle. High atomic number of $\mathrm{Cd}$ (48) and Te (52), higher electron density and high band gap $1.5 \mathrm{eV}$ predisposes CdTe to be a good material for direct conversion radiation detectors with good sensitivity and resolution. Apart from radiation detectors based on $\mathrm{Ge}$ or $\mathrm{Si}$, CdTe based detectors operate at room temperature without the necessity to be supercooled [1].

For high quality CdTe radiation detectors, the preparation of high-ohmic material with low concentration of trapping and recombination centers is necessary [2]. The native defects cadmium vacancy $\mathrm{V}_{\mathrm{Cd}}$ and tellurium anti-sity $\mathrm{Cd}_{\mathrm{Te}}$ are reported as the deep acceptor $(0.7-0.9$ $\mathrm{eV})$ and the deep donor (0.75 eV) [3], respectively. The presence of deep levels causes worse spectral resolution. Also, presence of unwanted minor charge carriers results in increased leakage currents and additive noise. However, the resistivity of available CdTe crystals for radiation detector applications is typically in the order of $10^{9} \Omega \mathrm{cm}$ and they suffer from a large leakage current when high bias voltages are applied. The most commonly adopted method to suppress the leakage current in a CdTe detector is to form Schottky barrier, using metal material contacts with high work function on the crystal [4]. To suppress deep levels, compensations by Indium/Chlorine dopants are used. Dopants are diffused inside the crystal by heating the crystal at a high temperature under high pressure. This results in creation donor-acceptor pairs of compensation admixtures with unwanted deep level impurities. The limiting factor is very fast diffusion of dopants into crystal bulk, which is difficult to control [5]. Furthermore, CdTe is very sensitive to high temperature processing, which leads to break of CdTe complex bonds and creation new defects.

Also, the contacting method is very important for overall detector performance. The most used technology, chemical deposition of gold contacts by aqueous solution of $\mathrm{AuCl}_{3}$ leads to defects creation in M-S interface area.

The subject of this paper is to investigate charge transport characteristics of analysed detectors and evaluate detectors noise. Two samples, a low-ohmic and a highohmic were investigated. Analysis of current transport properties was carried out by $I V$ characteristics analysis. To evaluate quality of detector noise spectral density was measured. All measurements were carried in the dark were carried out at the room temperature.

\section{THEORY}

On metal-semiconductor (M-S) interface originates energy barrier, denoting electrical and thermal properties of the junction structure. Presence of barriers is based on different work function of metal $\Phi_{\mathrm{m}}=E_{\mathrm{vac}}-E_{\mathrm{F} \text { (metal) }}$, and semiconductor $\Phi_{\mathrm{s}}=E_{\mathrm{vac}}-E_{\mathrm{F}(\text { semicon) }}$. Because of different work functions, M-S structure is heterogeneous. Further, we will consider gold as a metal contact material and ptype CdTe as a semiconductor material The Fermi level position is given by equation

$\mu_{\mathrm{F}}^{\prime}=\frac{E_{\mathrm{A}}}{2}-\frac{k T}{2} \ln \left(\frac{N_{\mathrm{A}}}{N_{\mathrm{V}}}\right)$,

where $\mu_{\mathrm{F}}^{\prime}$ is difference between the Fermi level $E_{\mathrm{F}}$ and the top of vallence band $E_{\mathrm{V}} E_{\mathrm{A}}$ is the acceptor energy level of localized states, $N_{\mathrm{A}}$ is the acceptor concentration, $N_{\mathrm{V}}$ is the effective density of states in the valence band $T$ is thermodynamic temperature and $k$ is the Boltzmann constant. $N_{\mathrm{V}}$ is given by

$N_{\mathrm{V}}=2\left(\frac{2 \pi m_{\mathrm{dh}} k T}{h^{2}}\right)^{\frac{3}{2}}$,

where $m_{\mathrm{dh}}=7.9 \cdot 10^{-31} \mathrm{~kg}$ is the density-of-state effective mass of the valence band. According to Eq. 3, for a p-type semiconductor, $N_{\mathrm{V}}=2.03 \cdot 10^{25} \mathrm{~m}^{-3}$. By solving equation (1) the Fermi level position is $0.25 \mathrm{eV}$, measured from the top of the valence band, or $E_{\mathrm{F}}=5.75 \mathrm{eV}$ measured from the bottom of the vacuum level with and $E_{\mathrm{A}}=0.3 \mathrm{eV}, N_{\mathrm{A}}$ $=5 \cdot 10^{21} \mathrm{~m}-3$ and $T=300 \mathrm{~K} \mathrm{[2]}$. So the value of the 
semiconductor work function is higher than the value of the metal work function. As the distance between the metal and the p-type semiconductor decreases, an increasing negative charge is built up at the metal surface. Connection between the semiconductor and the metal causes electrons flow from the metal surface, because $\phi_{\mathrm{m}}$ $=5.37 \mathrm{eV}$ is less than $\phi_{\mathrm{s}}=5.75 \mathrm{eV}$. This process will continue until Fermi levels in the metal and in the semiconductor are equal. This is shown in Fig. 1: When the gap between the metal and the semiconductor is small enough, electrons recombine with holes over the layer of length $L_{\mathrm{d}}$ in the bulk, because the hole concentration in the semiconductor is significantly less than the electron concentration in the metal surface.

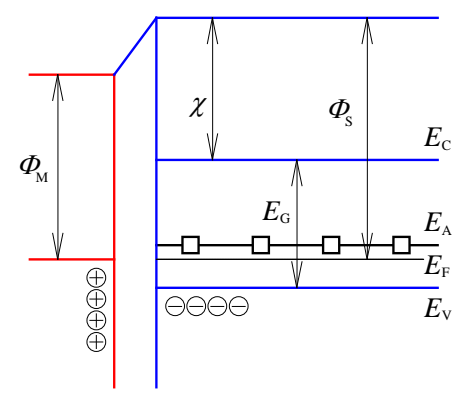

Fig. 1 Energy band diagram of Au and a p-type CdTe connection

All the p-type semiconductor energy levels bend within the depletion layer $L_{d}$ (Fig. 2). There are almost no free carriers over the depletion layer, so the resistance of the layer is significant. The barrier height is simply the difference between the metal work function and the semiconductor electron affinity. For an ideal contact between a metal and a p-type semiconductor [6], [7] the barrier high is given by

$e \Phi_{\mathrm{bp}}=E_{\mathrm{G}}-e\left(\Phi_{\mathrm{M}}-\chi\right)$

The diffusion potential, or built-in potential $V_{\mathrm{bi}}$, as shown in [7], for a p-type semiconductor equals to

$$
e V_{\text {bip }}=e \Phi_{\mathrm{S}}-e \Phi_{\mathrm{M}}=E_{\mathrm{G}}+e \chi-e \mu_{\mathrm{F}}^{\prime}-e \Phi_{\mathrm{M}} \text {. }
$$

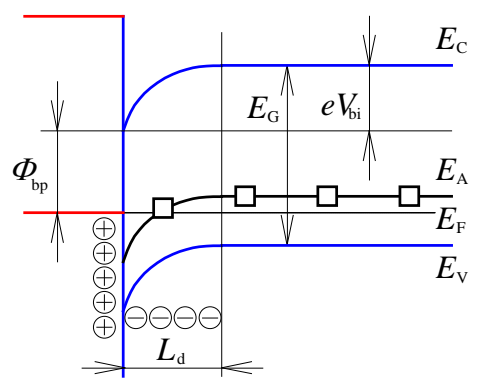

Fig. 2 Energy band diagram of Au and a p-type CdTe contact

For a p-type CdTe $V_{\text {bip }}=0.35 \mathrm{~V}$ according to equation (4). When an external voltage $V_{\text {ext }}$ is applied in reverse bias to the M-S junction, as shown in Fig. 3, the built-in potential is rising with applied voltage:

$V_{\mathrm{bir}}=V_{\mathrm{bi}}+V_{\mathrm{ext}}$, where $V_{\mathrm{bi}}$ is the built-in potential without applied electric field.

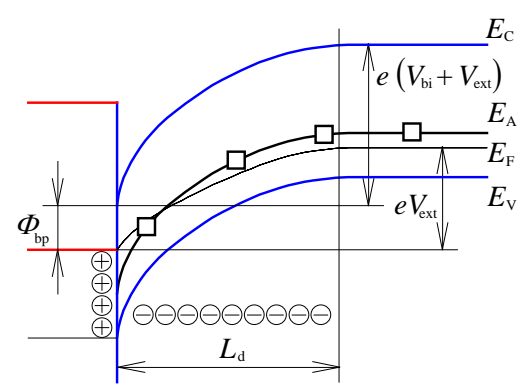

Fig. 3 Energy band diagram of Au and a p-type CdTe contact

If an external voltage $V_{\text {ext }}$ is applied in forward bias to the M-S junction, the built-in potential is lowering:

$V_{\mathrm{bif}}=V_{\mathrm{bi}}-V_{\mathrm{ext}}$.

With an applied external voltage in forward bias (negative potential is applied to the semiconductor) the semiconductor positive space charge within the depleted layer decreases and all the levels in the semiconductor rise by the value of $e V_{\text {ext }}$. With an applied external voltage to the sample one of the contacts operates in forward bias and another one operates in the reverse bias, so the barriers at each contact will be different. With an external voltage $V_{\text {ext }}>V_{\text {bi }}$ one of the barriers will disappear (at the forward bias contact) and there will be just one contact barrier in the sample. So we can imagine studied CdTe sample with golden contacts as a series connection of two Schottky diodes with a resistor between them.

A metal-semiconductor junction also results in an ohmic contact. Contacts show if the Schottky barrier height, is zero or negative. In such case, the carriers are free to flow in or out of the semiconductor so that there is a minimal resistance across the contact. For a p-type semiconductor, it requires that the work function of the metal must be close to or larger than the sum of the electron affinity and the bandgap energy. It is be problematic to find a metal that provides an ohmic contact to p-type semiconductors with a large bandgap such as CdTe. In case of CdTe, the ohmic contacts are based by increased concentration of charge carriers in semiconductor bulk, on leading to higher bulk conductivity and barrier thickening. This results in higher probability of carrier tunnelling through the potential barrier. So, Quantum-Mechanical tunnelling becomes the main charge transport mechanism through M-S interface.

\section{EXPERIMENTAL SETUP}

The measuring equipment intended for the CdTe detectors transport characteristics study is shown in Fig. 4. The sample with a load resistor is placed into the cryostat. The cryostat allows controlling operating temperature in the range from $77 \mathrm{~K}$ to $400 \mathrm{~K}$. The cryostat also acts as undesired electrical fields screening. The programmable DA converter Agilent E3631A is used for IV characteristics measurements. Separated measuring dots are interconnected by data acquisition unit Agilent 34970A with plug-in module Agilent 34902A, which is used for $\mathrm{AD}$ conversion and is connected with PC via GPIB bus. 


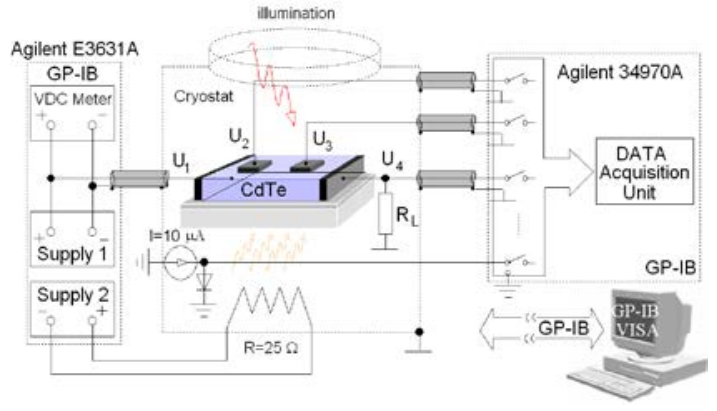

Fig. 4 Experimental setup for transport characteristics measurements

The noise voltage is transformed into the corresponding noise spectral density using FFT. Measured values were recorded and analysed in a PC. Noise spectral density measurements were conducted with applied voltage $U=25,50$ and $90 \mathrm{~V}$. both for the low-ohmic contact sample and for high-ohmic contact sample. The load resistance for the low-ohmic contacts sample $R_{\mathrm{L}}=10 \mathrm{k} \Omega$, for the high-ohmic sample $R_{\mathrm{L}}=10 \mathrm{M} \Omega$.

\section{MEASURING RESULTS AND DISCUSSION}

Result of $I V$ characteristics measurements for the lowohmic sample F33B8 is shown in Fig. 5. In Fig. 6, the $I V$ characteristic of the high-ohmic sample $\mathrm{B} 39 \mathrm{D} 1 \mathrm{H}$ is presented.

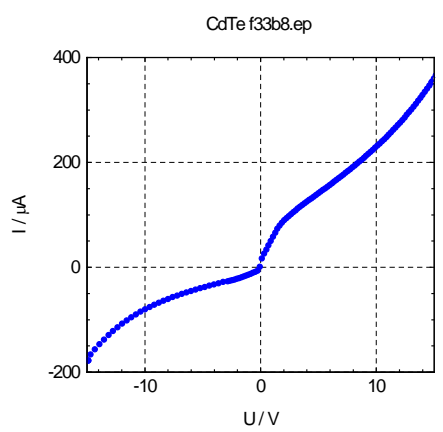

Fig. 5 IV characteristic of the low-ohmic sample F33B8

The high-ohmic p-type B39D1H sensor offers very good rectification properties as a result of the junction formed on the p-type high-resistivity crystal with low concentration of intrinsic charge carriers. At bias voltages after the full depletion of detector (approx. 1.2 V), characteristics show linear behaviour.

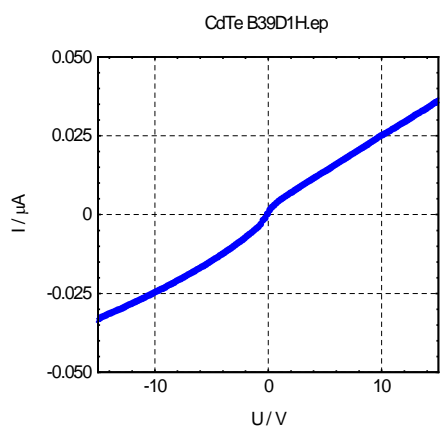

Fig. 6 IV characteristic of the high-ohmic sample B39D1H

In case of the low-ohmic sample, the characteristics should be symmetric, but from Fig. 6, the asymmetry shows differences in contact quality. Detector bulk has the same transport properties, for both biases, but the role of each contact area changes after changing voltage polarity. We can notice higher leakage current for negative bias of the sample. From the measured characteristics, we can conclude worse quality of contact that was reversely polarized when the detector was negatively biased. That caused formation of thicker depletion region that negatively affected quantum-mechanical tunnelling affectivity though potential barrier. Ideally, the $I V$ characteristics for ohmic contacts should be linear. The area of space charge always exists at the interface between two materials with different conductivity. The current flowing through these materials is the same. Therefore electric fields in these materials have to be different. As we can see from Poisson equation

$$
\frac{d^{2} \varphi}{d x^{2}}=\frac{\rho(x)}{\varepsilon_{0} \varepsilon}
$$

where $\varphi$ is the electric field potential, $\rho$ is the space charge concentration, $\varepsilon_{0}$ is permittivity of vacuum and $\varepsilon$ is relative permittivity, the space charge must exist when electric field changes. The carrier's concentration gradient and then diffusion currents are fundamentally connected with it. From this point of view there does not exist an ohmic contact (that fully agrees with Ohm's Law).

Noise spectral density analysis showed that in observed frequency range, the dominant noise source is $1 / f^{m}$ type. This type of noise prevails among whole analysed frequency range (The decline of signal PSD at frequencies over $300 \mathrm{~Hz}$ is caused by the signal filtration.). The low ohmic sample (Fig. 7) showed monotonous spectrum $1 / f^{m}$ with the slope $m$ close to unity.

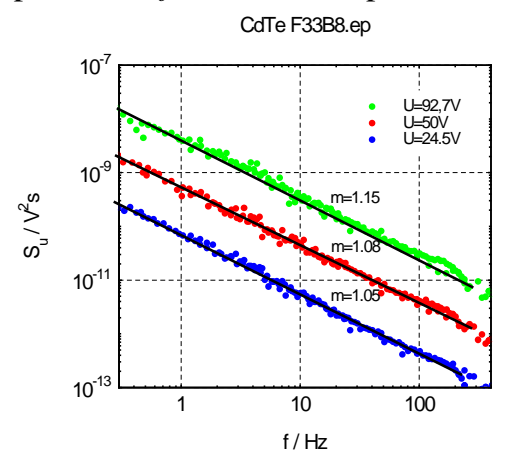

Fig. 7 Noise spectral density of the low-ohmic sample F33B8

On contrary, in case of high-ohmic sample frequency spectrum (Fig. 8), we can observe superposition of $1 / f$ noise with generation-recombination noise.

According to Hooge model, voltage noise spectral density is described by [8]

$S_{u}=\frac{\alpha U^{2}}{N f}$,

where $\alpha$ is material-specific Hooge constant, $N$ is total number of carriers. Voltage noise spectral density is proportional to the square of applied voltage.

From Fig. 9 and 10, the Hooge constant for our sample F33B8 were found by using Eq. (8). The sample volume is $V=0.152 \mathrm{~cm}^{3}$, the total number of charge carriers is $1.8 \times 10^{14}$ then Hooge constant $\alpha=5.5 \cdot 10^{-2}$. This value is higher than $\alpha_{\mathrm{H}}=2 \cdot 10^{-3}$ proposed by Hooge [8]. For high- 
ohmic sample B39D1H, the volume is $0.125 \mathrm{~cm}^{3}$, total number of carriers is $1.25 \cdot 10^{10}$. Hooge constant for this sample is $\alpha=4.4 \cdot 10^{-3}$. This value is very close to theoretical. This sample has good contacts. Nearly all noise origins in semiconductor bulk.

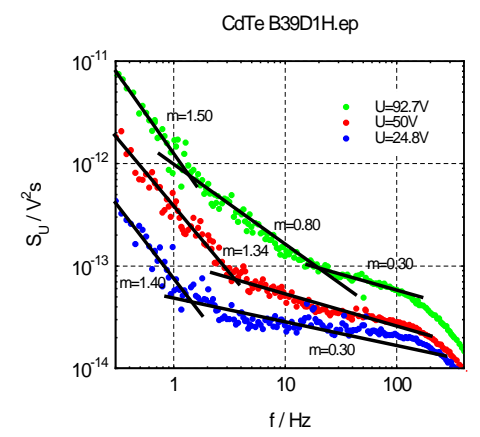

Fig. 8 Noise spectral density of the high-ohmic sample B39D1H

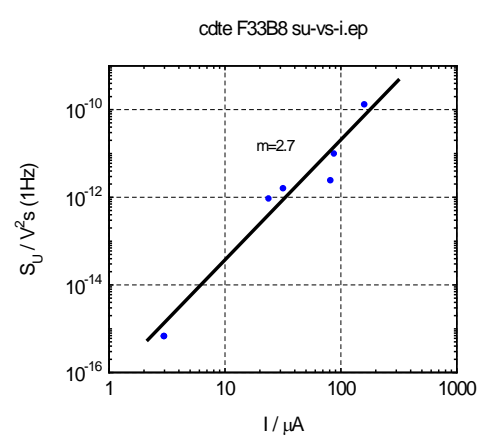

Fig. 9 Noise spectral density vs. applied voltage at $f=1 \mathrm{~Hz}$ of the low-ohmic sample F33B8

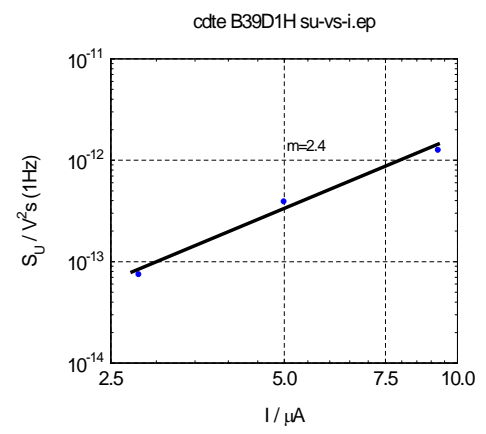

Fig. 10 Noise spectral density vs. applied voltage at $f=1 \mathrm{~Hz}$ of the semi-insulating sample B39D1H

We found that the sample F33B8 with ohmic contacts, shows contacts resistance asymmetry and measured noise correspond volume noise sources with submission of contacts noise. For ohmic contacts, charge carrier transport is supposed to happen by quantum-mechanical tunnelling, but due to existence of space charge in metalsemiconductor interface area, asymmetry appears. The symmetry of $I V$ characteristics showed nonlinearities for both analysed samples. Contacts of high-ohmic sample B39D1H showed good rectifying properties with same saturation current in both polarities. Comparing received material-specific constants in Hooges noise spectral density model, we can separate contacts submission to overall detector noise. Low-ohmic sample F33B8 has Hooges constant one order higher than theoretical, caused by contacts additional noise and its noise spectral density rises with exponent 2.7 (Fig. 9). The dominant source is contacts area. The low-ohmic sample, the $1 / f^{m}$ noise type with slope $m=1.05$ to 1.15 (depending on applied voltage) dominates at the whole spectrum. For high-ohmic sample, the Hooges close to theoretical value.. The noise spectral density is proportional to applied voltage with exponent 2.4 (Fig. 10). The slope of $1 / f^{m}$ noise parameter $m$ increases with applied voltage. Its value interval is from 1.4 to 1.5 . This is caused by superposition of more parallel $1 / f^{m}$ noise sources in the crystal bulk.

\section{REFERENCES}

[1] SZELES, C.: CdZnTe and CdTe materials for X-ray and gamma ray radiation detector applications. Physics State Solids. 2004, vol. 241, no. 3, p. 783-79, ISSN 1610-1634.

[2] TURKEVYCH, I. - GRIL, R. - FRANC, J. HÖSCHL, P. - BELAS, E-. MORAVEC, P. FIERDLE, M. - BENZ, K. W.: Preparation of semiinsulating CdTe doped with group IV elements by post growth annealing. In Crystal Research Technology 2003, 38, p. 288 - 296.

[3] HOFFMAN, D. M. - OMLING, P. - GRIMMEISS, H. G. - MEYER, B. K. - BENZ, K. W. SINERIUS, D.: Physical review Letters 1992. B45, 6247.

[4] MATSUMOTO, C. - TAKAHASHI, T. TAKIZAWA, K. - OHNO, R. - OZAKI, T. MORI, K.: IEEE Transactions on Nuclear Science 1998, 45, 428.

[5] HAGE-ALI, M. - SIFFERT, P.: Semiconductors for Room Temperature Nuclear Detector Applications In Semiconductors and Semimetals, 1995, Vol. 43: Academic Press, 1995), p. 275.

[6] SZE, S. M.: Physics of Semiconductor Device. John Wiley \& Sons New York, 1981, 832 pp.

[7] EPIFANOV, G. I.: Physical basis of microelectronics. Moscow, 1971, 376 p.

[8] HOOGE, F. N.: 1/f noise is no surface effect. Physics. Letters. 1969, A. 29, p.139-40.

Received February 8, 2013, accepted February 17, 2013

\section{BIOGRAPHIES}

Lubomír Grmela graduated in 1982 (MSc) at the Dept. of Radioelectronics of the Faculty of Electrical Engineering at Technical University in Brno. He defended his $\mathrm{PhD}$ in the field of stochastic processes in electronic devices in 1986. Since 1995 he is working as a tutor at the Dept. of Physics. His research focuses on nanodevices, optoelectronics characterisation and development.

Ondrej Šik graduated in 2009 at the Dept of Telecommunication Faculty of Electrical Engineering at Technical University in Brno. Currently, he is a postgradual student. The topic of his dissertation is Charge Transport properties of CdTe based radiation detectors. 